\title{
Association of genetic variation in COMT gene with pain related to sickle cell disease in patients from the walk-PHaSST study
}

This article was published in the following Dove Press journal: Journal of Pain Research

\author{
Yingze Zhang ${ }^{1,2}$ \\ Inna Belfer ${ }^{2,3}$ \\ Mehdi Nouraie' \\ Qilu Zeng' \\ Ruchika Goel ${ }^{4,5}$ \\ Yanxia Chu' \\ Inna Krasiy' \\ Lakshmanan Krishnamurti ${ }^{6,7}$
}

\section{On behalf of the walk- PHaSST Investigators and Patients}

'Division of Pulmonary, Allergy, and Critical Care Medicine, ${ }^{2}$ Department of Human Genetics, ${ }^{3}$ Department of Anesthesiology, University of Pittsburgh, ${ }^{4}$ Department of Pathology and Laboratory Medicine, ${ }^{5}$ Department of Pediatrics, Division of Pediatric Hematology/Oncology, Weill Cornell Medicine, ${ }^{6}$ Division of Hematology/Oncology, Children's Hospital of Pittsburgh, Pittsburgh, PA, ${ }^{7}$ AFLAC Center for Cancer and Blood Disorders, Children's Healthcare of Atlanta, Atlanta, GA, USA

Correspondence: Lakshmanan Krishnamurti

AFLAC Center for Cancer and Blood Disorders, Children's Healthcare of Atlanta, 2015 Uppergate Drive, Atlanta, GA 30322, USA

Tel + I 4047270710

Email lakshmanan.krishnamurti@emory. edu
Background: Vaso-occlusive pain episodes (VOEs) are the hallmark of sickle cell disease (SCD), and our current understanding of disease biology, treatment, and psychological covariates does not adequately explain the variability of pain in SCD. Functional variants in catechol-Omethyltransferase (COMT) gene contribute to variability in pain perception, but their impact on pain perception in African American SCD patients is not well known.

Methods: We studied COMT single-nucleotide polymorphisms (SNPs) rs6269, rs4633, rs4818, rs4680, and rs165599 to determine their relationship to patient self-reported pain, the number of acute VOEs, and their impact on daily life and health care utilization in 438 hemoglobin SS patients who participated in the walk-PHaSST study.

Results: In women, two risk SNPs (rs4633 and rs165599) and the corresponding haplotype (A $\underline{T C A} \underline{A}$ ) were associated with increased frequency of pain-related emergency room visit.

Conclusion: COMT functional variants may predispose SCD patients to worse acute pain in women. The association of COMT variants with the intensity of self-reported acute pain warrants further genetic study of pain perception in SCD.

Keyword: ER visit, catechol-O-methyltransferase, haplotype, VOE, SNP

\section{Introduction}

Vaso-occlusive pain is the hallmark of sickle cell disease (SCD). Vaso-occlusive pain episodes (VOEs) vary in frequency, duration, and severity between and within patients. ${ }^{1,2}$ SCD-associated organ and tissue damages, such as avascular necrosis, also contribute to pain. ${ }^{1,2}$ Similar to other human pain conditions, interindividual pain experiences in patients with SCD vary considerably. Although factors related to disease biology, treatment, and patient psychological factors may explain a part of this variability, genetic factors may also contribute to susceptibility to painful disorders and may modulate the perception of pain by individual patients. ${ }^{3}$

Catechol- $O$-methyltransferase (COMT) is a key enzyme in the catabolic pathway of pain-relevant neurotransmitters such as dopamine, epinephrine, and norepinephrine and plays a significant role in the perception of pain. ${ }^{4-6}$ The enzymatic activity of COMT is partly determined by genetic factors. ${ }^{7}$ Functional COMT variants are associated with interindividual differences in clinical pain experience, pain sensitivity to experimental stimulation, and response to analgesics. ${ }^{3,6}$ The best known genetic variant in COMT is a single-nucleotide polymorphism (SNP), rs4680, also known as Val158Met. ${ }^{6,8}$ The substitution of valine (Val) for methionine (Met) at position 158 of the soluble form of COMT leads to a three- to fourfold reduction in enzyme activity. ${ }^{5,7}$ Among 
healthy subjects, homozygous Met/Met individuals showed diminished regional $\mu$-opioid system responses to pain compared with heterozygous individuals when hypertonic saline was injected into the masseter muscles. ${ }^{6}$ In White Americans, three major COMT haplotypes corresponding to high pain sensitivity (HPS), average pain sensitivity (APS), and low pain sensitivity (LPS) have been reported..$^{5,7}$ These haplotypes were based on rs4680, two additional coding SNPs (rs4633 and rs4818), and a promoter SNP (rs6269) of the COMT gene. In a cell model, these haplotypes are associated with up to 20-fold differences in enzyme activity with LPS and HPS having the highest and lowest activities, respectively. ${ }^{5,7}$ Functional variations in COMT are associated with the risk of developing chronic pain syndromes, ${ }^{9}$ postsurgical pain, ${ }^{10}$ and analgesia. ${ }^{4}$ Recently, specific effects of stress and gender on COMT variants and pain perception have been reported. ${ }^{11}$

Comprehensive analysis of COMT genetic variants and their role in pain experienced by patients with SCD is limited. Furthermore, the distribution of COMT haplotypes in African American (AA) populations is not well known. In the current study, we determined COMT haplotypes based on five SNPs in a large cohort of AA patients with SCD and evaluated the association between COMT functional variants and haplotypes and self-reported pain episodes in the year and month prior to study enrollment.

\section{Methods}

\section{Study cohort and clinical characterization}

Subjects with hemoglobin SS genotypes (HbSS) from the screening phase of the walk-PHaSST, an NHLBI-sponsored study to screen patients with SCD for pulmonary hypertension (PH) and offer participation in a randomized control study of sildenafil, were included in this study. ${ }^{12,13}$ The Institutional Review Board of the University of Pittsburgh and that of all participating institutes of the walk-PHaSST study approved this study. Investigators from each clinical site obtained written informed consent from all the participants. Subjects were evaluated by self-reported history modeled after the National Institute of Heath Pulmonary Hypertension screening survey, ${ }^{14}$ physical examination, laboratory screening, and transthoracic Doppler echocardiography. Exclusion criteria included current pregnancy or lactation, any stroke within the last 6 weeks, and diagnosis of pulmonary embolism within the last 3 months.

\section{Self-reported pain phenotype}

At the screening visit, patients completed a health history survey, which included a self-description of acute SCD pain and listed associated treatments. Subjects provided detailed medical history including the total number of episodes of acute pain in the month and the year before screening. Acute pain was classified as 1) mild (may or may not require pain medicine and did not prevent normal daily activity), 2) moderate (required medications and caused significant changes in everyday activities), 3) severe (patient went to the emergency room [ER] but was not admitted), and 4) extremely severe (required hospital admission).

\section{DNA isolation and genotyping}

Genomic DNA was isolated from blood using the QIAamp DNA isolation kit (Qiagen NV, Venlo, the Netherlands). Five SNPs of the COMT gene, ie. rs6269, rs4633, rs4818, rs4680, and rs165599, were genotyped using TaqMan platform with predesigned primer and probes and 7900 DNA analyzer (ABI, Foster City, CA, USA).

\section{Statistical analysis}

We calculated site-specific allele frequencies using genotype counting and tested for departures from Hardy-Weinberg equilibrium using goodness of fit statistics. Pairwise estimates of linkage disequilibrium (LD) were measured as $D^{\prime}$ and $r^{2}$ from the diploid data. ${ }^{15}$ Haplotype analysis was conducted using PHASE (Version 2.1) ${ }^{16}$ to infer haplotypes from the unphased SNPs and coding the resulting haplotypes according to the number of copies present for each participant. Genetic association of the COMT variants with chronic pain was analyzed for both the presence and the intensity experienced by patients. For the pain intensity analysis, only subjects who reported positive pain scores were used for the association analysis. We assessed the association between the number of severe and extremely severe pain episodes and SNP or haplotype in a General Linear Model. Analyses were performed using SAS Version 9.3 (SAS Institute Inc., Cary, NC, USA). Additionally, the gender-specific analysis was conducted. Bonferroni correction was performed for significant associations to adjust for multiple comparisons. A $P$-value of $<0.01$ was considered significant for the $\operatorname{SNP}(0.05 / 5=0.01)$ and the haplotype $(0.05 / 5=0.01)$-specific analysis.

\section{Results}

\section{Self-reported pain and associated clinical factors}

The median age of the 438 patients was 36 years (1269 years), and 51.8\% were females (Table 1). Opioids and hydroxyurea usage were listed for 32.2 and $44.3 \%$ of the subjects at enrollment. Subjects who were on opioid treatment had more ER visits (severe and/or extremely severe 
Table I Demographics, opioid, and hydroxyurea treatment and association with pain-related ER visit in the past year (HbSS, $\mathrm{n}=438$ )

\begin{tabular}{llll}
\hline Characteristics & Results & $\begin{array}{l}\text { Effect on the } \\
\text { number } \\
\text { of pain episodes }\end{array}$ \\
\cline { 2 - 4 } & & Beta & P-value $^{\mathbf{2}}$ \\
\hline Age, mean (SD) (years) & $36.0(12.5)$ & -0.02 & 0.548 \\
Female gender & $227(51.8)$ & 0.44 & 0.662 \\
Current opioid use & $141(32.2)$ & 4.07 & $<0.00$ I \\
Current hydroxyurea use & $194(44.3)$ & 1.92 & 0.062 \\
Acute pain in the past month & & & \\
Mild & $222(51.1)$ & N/A & N/A \\
Moderate & $194(44.5)$ & N/A & N/A \\
Severe & $74(16.9)$ & N/A & N/A \\
Extremely severe & $62(14.2)$ & N/A & N/A \\
Acute pain in the past year ${ }^{\mathrm{b}}$ & $283(65.5)$ & N/A & N/A \\
Mild & $296(68.2)$ & N/A & N/A \\
Moderate & $197(45.0)$ & N/A & N/A \\
Severe & $232(53.0)$ & N/A & N/A \\
Extremely severe &
\end{tabular}

Notes: Results are in $\mathrm{n}(\%)$ unless otherwise specified. ${ }^{\mathrm{a}} \mathrm{From}$ a General Linear Model with number of severe and/or extremely severe pain episodes (pain-related ER visit) in the past year as outcome. 'The number of subjects (n) who reported greater than zero of each specific type of acute pain in the month or year before enrollment in the study. The Beta and $P$-value with significances are in bold. Abbreviations: HbSS, hemoglobin SS genotypes; ER, emergency room; N/A, not applicable.

pain episodes) in the year before screening than subjects who were not. This association remained significant after adjusting for age and gender $(P<0.001)$. Hydroxyurea usage, age, and gender appeared unrelated to the number of episodes of severe and/or extremely severe pain in the year before study enrollment.

\section{Genotype and haplotype frequencies of COMT variants}

The distribution of rs6269, rs4633, rs4818, rs4680, and rs165599 followed Hardy-Weinberg equilibrium $(P \geq 0.01)$, with minor allele frequencies of 37.4, 30.5, 19.4, 28.6, and $28.1 \%$, respectively (Table 2). Allele frequencies for these SNPs and degrees of LD among them were similar to those from the 1000 Genomes Project (http://browser.1000genomes. org/index.html) for the African (AFR) population. Except for rs6269, the European (EUR) population had much higher minor allele frequencies compared to AFR. Higher LD was observed for rs6269, rs4633, rs4818, and rs4680 in EUR than in AFR and AA populations from this study (Figure 1). The rs 165599 had lower LD with any of the other SNPs in all populations. Nine haplotypes with the frequencies of $>3 \%$ accounted for $97.6 \%$ of all haplotypes in our cohort (Table 2). HT2 was unique to this AA SCD cohort, and $34.2 \%$ of the subjects carried one or two copies of this haplotype.

\section{Gender-specific allele association with more pain-related ER visits in the past month}

To determine whether the COMT variants are associated with acute VOEs that resulted in health care utilization, we first analyzed the association of COMT SNPs with the presence and frequencies of pain-related ER visit in the past month and year. Since both severe and extremely severe pain episodes are defined by an ER visit, subjects are more likely able to recall them. SNP frequencies were not significantly different between the group who did, versus did not, have pain-related ER visits in the past month or year before study enrollment. Among the subjects $(n=111)$ who experienced at least one ER visit in the past month or year, homozygotes of rs 4633 or rs 165599 common alleles experienced far fewer episodes of ER visit compared to carriers of the rare alleles for each of these SNPs, in the month before screening (Table 3). A gender-stratified analysis demonstrated that these associations were only in females for both SNPs and remained significant for rs 165599 after SNP and gender multiple comparison correction $(P<0.005)$. Adjusting for current opioid treatment did not change these results. None of the SNPs were associated with the episodes of pain-related ER visit in the past year.

\section{Gender-specific haplotype association with more pain-related ER visits in the past month}

To determine whether the haplotypes containing the risk alleles of rs4633 and rs165599 impact the episodes of painrelated ER visit in the previous month, we analyzed the association between the five common haplotypes with $>5 \%$ frequencies (HT1-HT5) and this phenotype. Carriers of HT3 (A $\underline{T C A} \underline{A}$ ), which contains the risk alleles of both rs 4633 and rs165599, had the most episodes of pain-related ER visit compared to noncarrier subjects ( 3.28 vs $1.80, P=0.0004$ ), and this effect was more profound in females (4.06 vs 1.82, $P=0.0001$ ) (Table 4). After adjusting for the current opioids treatment, these $P$-values did not change. Compared to the associations of each individual SNP, the HT3 haplotype association was much stronger, suggesting a synergistic effect of these SNPs. Therefore, we designate the HT3 as a "risk haplotype" for VOE (acute pain resulted in ER visit) in female AA SCD patients.

\section{Discussion}

We present the first comprehensive report on the association of functional COMT variants and haplotypes with 
Table 2 Allele and genotype frequencies of COMT SNPs and haplotypes in subjects with HbSS genotype from the walk-PHaSST study

\begin{tabular}{|c|c|c|c|c|c|c|}
\hline \multirow[t]{2}{*}{ SNP } & \multicolumn{3}{|c|}{ Genotype frequency (\%) } & \multicolumn{3}{|c|}{ Minor allele frequency $(\%)^{a}$} \\
\hline & $\overline{M M}$ & Mm & $\mathbf{m m}$ & $\overline{A A}$ & AFR & EUR \\
\hline rs6269 (A/G) & 40.0 & 45.2 & 14.8 & 37.4 & 36.0 & 40.4 \\
\hline rs4633 (C/T) & 46.2 & 46.5 & 7.3 & 30.5 & 32.7 & 51.7 \\
\hline rs48I8 (C/G) & 64.6 & 32.0 & 3.4 & 19.4 & 16.9 & 39.7 \\
\hline rs4680 (G/A) & 49.7 & 43.5 & 6.9 & 28.6 & 30.9 & 51.6 \\
\hline rsI65599 (G/A) & 52.8 & 38.3 & 8.9 & 28.1 & 26.4 & 69.1 \\
\hline \multirow[t]{2}{*}{ Haplotype (>3\%) } & \multicolumn{3}{|c|}{ Diplotype frequency (\%) } & Haplotype & \multirow{2}{*}{\multicolumn{2}{|c|}{$\begin{array}{l}\text { Pain responsive haplotype in } \\
\text { American Caucasianc }\end{array}$}} \\
\hline & $\overline{0}$ & I & 2 & frequency $(\%)^{b}$ & & \\
\hline HTI (ACCGG) & 55.7 & 39.7 & 4.6 & 24.4 & \multicolumn{2}{|l|}{ HPS } \\
\hline HT2 (GCCGG) & 65.8 & 30.8 & 3.4 & 18.8 & \multicolumn{2}{|c|}{ Not reported in Caucasian } \\
\hline HT3 (ATCAA) & 68.7 & 28.8 & 2.5 & 16.9 & \multicolumn{2}{|c|}{ APS } \\
\hline HT4 (GCGGG) & 76.7 & 22.8 & 0.5 & 11.9 & \multicolumn{2}{|l|}{ LPS } \\
\hline HT5 (ATCAG) & 81.1 & 18.0 & 0.9 & 9.9 & \multicolumn{2}{|l|}{ APS } \\
\hline HT6 (ACCGA) & 91.8 & 7.8 & 0.5 & 4.3 & \multicolumn{2}{|l|}{ LPS } \\
\hline HT7 (GCGGA) & 91.8 & 7.8 & 0.5 & 4.3 & \multicolumn{2}{|l|}{ HPS } \\
\hline HT8 (ATCGG) & 92.5 & 7.5 & 0 & 3.8 & & \\
\hline HT9 (ACGGG) & 93.6 & 6.4 & 0 & 3.2 & & \\
\hline
\end{tabular}

Notes: ${ }^{a}$ Minor allele definition based on current study $(n=438)$. AA population was based on current study; AFR and EUR populations were based on 246 and 379 subjects from the 1000 Genomes Project (http://www. I000genomes.org/). ${ }^{24}$ bThe haplotype frequency was calculated by counting each specific haplotype and calculating the percentage of it based on the total haplotype count $(2 \times 438)$ in the 438 subjects of this study. ${ }^{\mathrm{T}}$ The HPS, APS, and LPS haplotypes were based on the definition by Diatchenko et al for American Caucasian. ${ }^{5}$

Abbreviations: AA, African American; AFR, African; APS, average pain sensitivity; COMT, catechol-O-methyltransferase; EUR, European; HbSS, hemoglobin SS genotypes; HPS, high pain sensitivity; LPS, low pain sensitivity; SNPs, single-nucleotide polymorphisms.

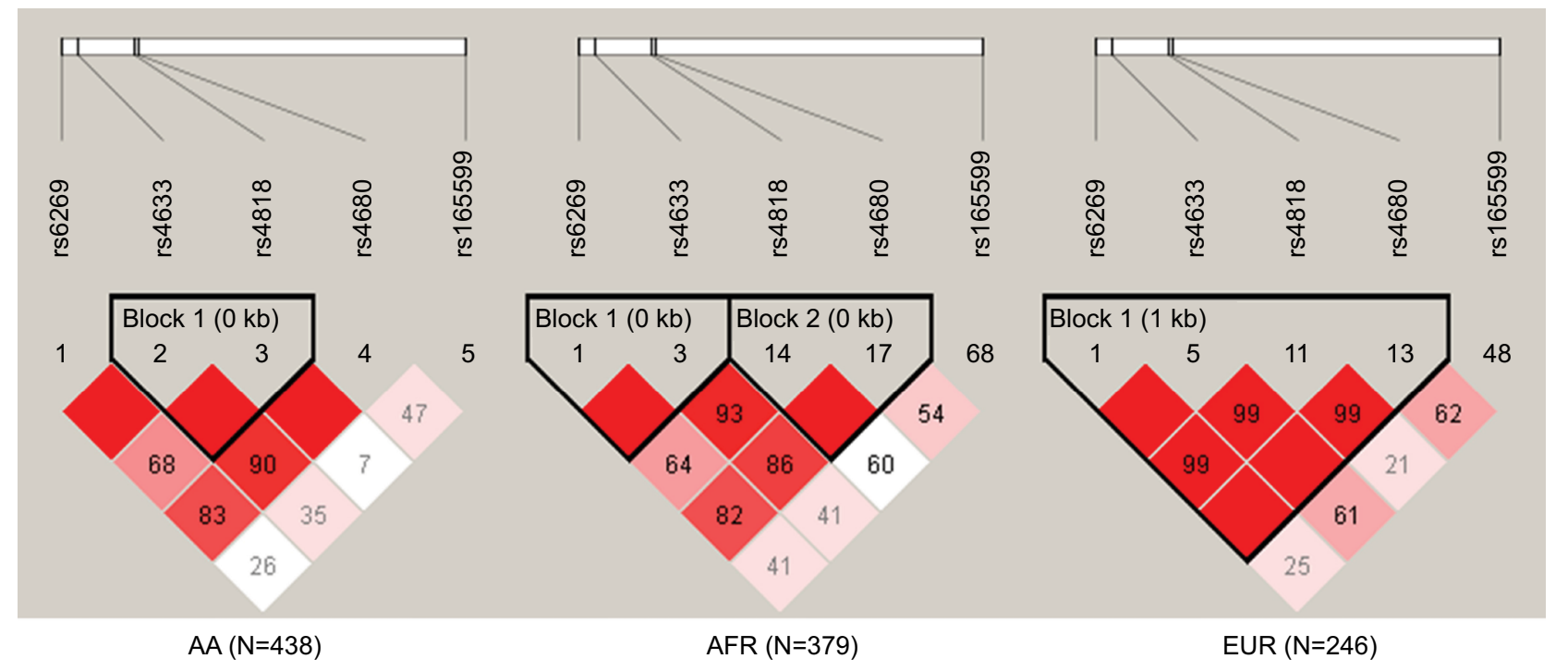

Figure I Haplotype structure of the COMT variants.

Notes: Haplotype structure was derived using Haploview. AA was based on current study. AFR and EUR were based on available data from the I000 Genome Project (http:// www. 1000 genomes.org/). AFR included populations of Yoruba in lbadan, Nigeria (YRI), Luhya in Webuye, Kenya (LWK), and Americans of African ancestry in southwest USA (ASW). EUR included populations of America Utah residents with Northern and Western European ancestry from the CEPH collection (CEU), Toscani in Italia (TSI), Finnish in Finland (FIN), British in England and Scotland (GBR), Iberian populations in Spain (IBS).

Abbreviations: AA, African American; AFR, African; COMT, catechol-O-methyltransferase; EUR, European.

self-reported pain-related ER visits and health care utilizations in a large multicenter AA cohort of SCD patients. The number of episodes of pain-related ER visits was associated with current opioid use. The main finding of the effects of COMT variants in this SCD cohort was the association of rs4633 and rs165599 with the frequency of pain-related ER visit in the month prior to study enrollment. Additionally, we observed a strong haplotype effect on the same phenotype and identified a risk haplotype for acute pain, which resulted in health care utilization for VOEs in female patients from this AA SCD cohort.

Jhun et $a 1^{17}$ reported that the functional COMT SNP rs4680 A allele for $158 \mathrm{Met}$ was associated with a higher health care utilization than the Val allele in a small SCD 
Table 3 Gender-specific association of COMT SNPs with the frequency of pain-related ER visit in the past month

\begin{tabular}{|c|c|c|c|c|c|c|}
\hline \multirow[t]{2}{*}{ SNP } & \multirow[t]{2}{*}{$\mathbf{N}$} & \multirow[t]{2}{*}{ Genotype (N) (CC/CT/TT) } & \multicolumn{3}{|c|}{ Genotype mean (SE) } & \multirow[t]{2}{*}{$P$-value* } \\
\hline & & & CC & CT & TT & \\
\hline \multicolumn{7}{|l|}{ rs4633 } \\
\hline All & 110 & $56 / 48 / 6$ & $1.80(0.17)$ & $2.54(0.37)$ & $3.50(1.38)$ & 0.016 \\
\hline Female & 66 & $34 / 29 / 3$ & $1.79(0.22)$ & $2.86(0.52)$ & $5.00(2.64)$ & 0.007 \\
\hline \multirow[t]{2}{*}{ Male } & 44 & $22 / 19 / 3$ & $1.81(0.25)$ & $2.05(0.49)$ & $2.00(0.58)$ & 0.689 \\
\hline & & Genotype (N) (GG/GA/AA) & GG & GA & AA & \\
\hline \multicolumn{7}{|c|}{ rs165599 } \\
\hline All & 111 & $67 / 35 / 9$ & $1.79(0.15)$ & $2.86(0.44)$ & $3.00(1.32)$ & 0.009 \\
\hline Female & 67 & $42 / 19 / 6$ & $1.83(0.19)$ & $3.21(0.65)$ & $4.00(1.90)$ & 0.004 \\
\hline Male & 44 & $25 / 16 / 3$ & $1.72(0.23)$ & $2.44(0.56)$ & $1.00(0)$ & 0.713 \\
\hline
\end{tabular}

Note: $*$ The $P$-values were calculated based on an additive model of the specific risk alleles and $P$-values with significance are in bold.

Abbreviations: COMT, catechol-O-methyltransferase; ER, emergency room; SE, standard error, SNPs, single-nucleotide polymorphisms.

Table 4 Gender-specific association of COMT haplotype with the frequency of pain-related ER visit in the past month

\begin{tabular}{|c|c|c|c|c|c|c|}
\hline \multirow[t]{2}{*}{ Haplotype $^{a}$} & \multirow[t]{2}{*}{$\mathbf{n}$} & \multirow{2}{*}{$\begin{array}{l}\text { Haplotype } \\
\text { (n) }(0 / 1+2 \text { copies })\end{array}$} & \multicolumn{2}{|c|}{ Haplotype mean (SE) } & \multirow[t]{2}{*}{$P$-value* } & \multirow[t]{2}{*}{ Risk allele (n) } \\
\hline & & & 0 copy & $I+2$ copies & & \\
\hline HTI (ACCGG) & & & & & & 0 \\
\hline All & 111 & $57 / 54$ & $2.25(0.29)$ & $2.20(0.28)$ & 0.916 & \\
\hline Female & 67 & $33 / 34$ & $2.61(0.46)$ & $2.24(0.34)$ & 0.517 & \\
\hline Male & 44 & $24 / 20$ & $\mathrm{I} .75(0.2 \mathrm{I})$ & $2.15(0.48)$ & 0.421 & \\
\hline HT2 (GCCGG) & & & & & & 0 \\
\hline All & 111 & $70 / 41$ & $2.49(0.30)$ & $1.78(0.15)$ & 0.083 & \\
\hline Female & 67 & $40 / 27$ & $2.90(0.45)$ & $1.70(0.19)$ & 0.034 & \\
\hline Male & 44 & $30 / 14$ & $1.93(0.34)$ & $1.92(0.27)$ & 0.993 & \\
\hline HT3 (ATCAA) & & & & & & 2 \\
\hline All & 111 & $79 / 32$ & $1.80(0.13)$ & $3.28(0.57)$ & 0.0004 & \\
\hline Female & 67 & $49 / 18$ & $1.82(0.17)$ & $4.06(0.85)$ & 0.0001 & \\
\hline Male & 44 & $30 / 14$ & $\mathrm{I} .77(0.2 \mathrm{I})$ & $2.29(0.64)$ & 0.327 & \\
\hline HT4 (GCGGG) & & & & & & 0 \\
\hline All & 111 & $83 / 28$ & $2.34(0.25)$ & $1.89(0.26)$ & 0.330 & \\
\hline Female & 67 & $50 / 17$ & $2.52(0.36)$ & $2.11(0.39)$ & 0.540 & \\
\hline Male & 44 & $33 / 11$ & $2.06(0.3 I)$ & $1.55(0.28)$ & 0.366 & \\
\hline HT5 (ATCAG) & & & & & & I \\
\hline All & 111 & $90 / 21$ & $2.27(0.22)$ & $2.05(0.43)$ & 0.666 & \\
\hline Female & 67 & $53 / 14$ & $2.43(0.32)$ & $2.36(0.63)$ & 0.913 & \\
\hline Male & 44 & $37 / 7$ & $2.03(0.29)$ & $\mathrm{I} .43(0.30)$ & 0.375 & \\
\hline
\end{tabular}

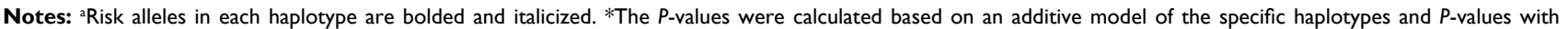
significance are in bold.

Abbreviations: COMT, catechol-O-methyltransferase; ER, emergency room; SE, standard error.

cohort. Our results confirm and extend their findings since the rs4680A is a part of the risk haplotype (HT3) and may also contribute to the synergistic SNP effect in the strong haplotype associations with pain-related ER visit.

Overall acute pain reflects the total burden of pain in SCD patients. ${ }^{1}$ Since recall is extremely unreliable beyond 1 month and to avoid memory bias, we considered pain intensity in the month prior to screening to be a good marker of recent acute pain or acute exacerbation of chronic pain. We only analyzed severe and extremely severe pain as these episodes required an ER visit, which improves the validity of patient's recall. The female-specific association identified for
COMT variants is in line with growing evidence that certain genes contribute differently to pain perception and sensitivity in men and women. ${ }^{18}$ Moreover, COMT variants have demonstrated generally more profound effects in females both in mice and humans. ${ }^{19}$ Putative mechanisms of COMT gender specificity include its regulation by estrogen and the differences in physiological responses to stressors between the genders. ${ }^{19}$ In contrast, presentation of pain related to SCD-specific factors differed between genders in clinical studies; for instance, women with SCD were shown to have a higher rate of VOEs. ${ }^{2}$ A recent study also revealed that GCH1 haplotype association with pain crisis was restricted 
to females with SCD. ${ }^{20}$ Further studies are indicated to determine if independent and/or combined effects of COMT and $G C H 1$ functional alleles influence pain phenotypes in females with SCD. Because COMT variations affect both pain and analgesia, future prospective studies monitoring SCD patients before and during hospitalization are also warranted to investigate individual effects of COMT SNPs and haplotypes and their interactions with $\mu$-opioid receptor (OPRM1) variants on opioid treatment outcomes.

We describe here, for the first time, the COMT functional haplotype distribution in AA SCD patients. The new haplotype (HT2) that was unique for AA supports population variations in the COMT locus. For example, Ittiwut et $\mathrm{al}^{21}$ described a three-allele COMT haplotype associated with cocaine-induced paranoia in AA patients, while another COMT haplotype was associated with the same phenotype in White patients. This evidence may explain racial differences in erythrocyte COMT activity in AA, and White patients in that higher enzymatic activity in AA patients could be determined by functional alleles in $C O M T^{22}$

The strengths of our study are that we used a large cohort of well-characterized patients who are hemoglobin SS genotype and incorporate the impact of pain on daily activities, such as work, into the definition of pain severity. However, this study had several limitations. We relied on patient self-reported ER visits and did not seek any independent verification of patient's health care utilization at various time points. We did not capture data on the moods or psychological states of these patients. This study, as other studies of pain in SCD patients, suffers from a lack of validated instruments to capture SCD pain descriptors reliably, in real time and in the patient's natural environment. We have recently developed and validated an electronic pain diary for the capture of SCD pain phenotypes in real time for use in clinical and translational research. ${ }^{23}$ Instruments such as this may be useful in defining pain phenotype defined with accuracy and granularity in studies of genetic determinants of pain perception in SCD.

The association of COMT functional variants and haplotypes with patient self-reported pain phenotypes in SCD observed in this study provides the rationale for future studies to better understand the genetic regulation of pain sensitivity in AA SCD patients. Additional studies with longitudinal design and comprehensive pain assessment will help to better characterize pain phenotypes for genotype-phenotype associations. Such studies have the potential to provide insights into the molecular mechanisms associated with SCD pain and, ultimately, lead to better prevention and management of pain related to SCD.

\section{Acknowledgments}

We thank Dr Mark Gladwin and the investigators of the walk-PHaSST study and the patients who participated in the study. We also thank the walk-PHaSST clinical site team: Albert Einstein; College of Medicine: Jane Little and Verlene Davis; Columbia University: Robyn Barst, Erika Rosenzweig, Margaret Lee, and Daniela Brady; UCSF Benioff Children's Hospital Oakland: Claudia Morris, Ward Hagar, Lisa Lavrisha, Howard Rosenfeld, and Elliott Vichinsky; Children's Hospital of Pittsburgh of UPMC: Regina McCollum; Hammersmith Hospital, London: Sally Davies, Gaia Mahalingam, Sharon Meehan, Ofelia Lebanto, and Ines Cabrita; Howard University: Victor Gordeuk, Oswaldo Castro, Onyinye Onyekwere, Vandana Sachdev, Alvin Thomas, Gladys Onojobi, Sharmin Diaz, Margaret Fadojutimi-Akinsiku, and Randa Aladdin; Johns Hopkins University: Reda Girgis, Sophie Lanzkron, and Durrant Barasa; NHLBI: Mark Gladwin, Greg Kato, James Taylor, Wynona Coles, Catherine Seamon, Mary Hall, Amy Chi, Cynthia Brenneman, Wen Li, and Erin Smith; University of Colorado: Kathryn Hassell, David Badesch, Deb McCollister, and Julie McAfee; University of Illinois at Chicago: Dean Schraufnagel, Robert Molokie, George Kondos, Patricia Cole-Saffold, and Lani Krauz; and National Heart \& Lung Institute, Imperial College London: Simon Gibbs. The authors also thank the data coordination center team from Rho, Inc., Nancy Yovetich, Rob Woolson, Jamie Spencer, Christopher Woods, Karen Kesler, Vickie Coble, and Ronald W Helms, and specially thank the volunteers who participated in the Walk-PHaSST study. This project was funded with federal funds from the NHLBI, NIH, Department of Health and Human Services, under contract HHSN268200617182C. This study is registered at www. clinicaltrials.gov as NCT00492531. We thank Ms Christine Heiner, Scientific Writer at the University of Pittsburgh Department of Anesthesiology/Department of Surgery, for her kind help with editing of this article.

\section{Author contributions}

YZ, IB, and LK conceptualized the study. YC and IK performed DNA isolation and genotyping. LK and the walkPHaSST Investigators recruited subjects and collected clinical phenotypes and blood samples. YZ and YC were involved in sample banking. YZ, MN, QZ, and RG analyzed the data. YZ, 
IB, and LK drafted and finalized the article. All of the authors critically reviewed the results. All authors contributed toward data analysis, drafting and critically revising the paper and agree to be accountable for all aspects of the work.

\section{Disclosure}

The authors report no conflicts of interest in this work.

\section{References}

1. Ballas SK, Gupta K, Adams-Graves P. Sickle cell pain: a critical reappraisal. Blood. 2012;120(18):3647-3656.

2. Platt OS, Thorington BD, Brambilla DJ, et al. Pain in sickle cell disease. Rates and risk factors. N Engl J Med. 1991;325(1):11-16.

3. Young EE, Lariviere WR, Belfer I. Genetic basis of pain variability: recent advances. J Med Genet. 2012;49(1):1-9.

4. Belfer I, Segall S. COMT genetic variants and pain. Drugs Today (Barc). 2011;47(6):457-467.

5. Diatchenko L, Nackley AG, Slade GD, et al. Catechol-O-methyltransferase gene polymorphisms are associated with multiple pain-evoking stimuli. Pain. 2006;125(3):216-224.

6. Zubieta JK, Heitzeg MM, Smith YR, et al. COMT val158met genotype affects mu-opioid neurotransmitter responses to a pain stressor. Science. 2003;299(5610):1240-1243.

7. Nackley AG, Shabalina SA, Tchivileva IE, et al. Human catechol-Omethyltransferase haplotypes modulate protein expression by altering mRNA secondary structure. Science. 2006;314(5807):1930-1933.

8. Tammimaki A, Mannisto PT. Catechol-O-methyltransferase gene polymorphism and chronic human pain: a systematic review and metaanalysis. Pharmacogenet Genomics. 2012;22(9):673-691.

9. Cohen H, Neumann L, GlazerY, Ebstein RP, Buskila D. The relationship between a common catechol-O-methyltransferase (COMT) polymorphism val(158) met and fibromyalgia. Clin Exp Rheumatol. 2009;27(5 Suppl 56):S51-S56.

10. Dai F, Belfer I, Schwartz CE, et al. Association of catechol-O-methyltransferase genetic variants with outcome in patients undergoing surgical treatment for lumbar degenerative disc disease. Spine J. 2010;10(11):949-957.

11. Meloto CB, Bortsov AV, Bair E, et al. Modification of COMT-dependent pain sensitivity by psychological stress and sex. Pain. 2016;157(4): 858-867.
12. Sachdev V, Kato GJ, Gibbs JS, et al. Echocardiographic markers of elevated pulmonary pressure and left ventricular diastolic dysfunction are associated with exercise intolerance in adults and adolescents with homozygous sickle cell anemia in the United States and United Kingdom. Circulation. 2011;124(13):1452-1460.

13. Machado RF, Barst RJ, Yovetich NA, et al. Hospitalization for pain in patients with sickle cell disease treated with sildenafil for elevated TRV and low exercise capacity. Blood. 2011;118(4):855-864.

14. Gladwin MT, Sachdev V, Jison ML, et al. Pulmonary hypertension as a risk factor for death in patients with sickle cell disease. $N$ Engl J Med. 2004;350(9):886-895.

15. Devlin B, Risch N. A comparison of linkage disequilibrium measures for fine-scale mapping. Genomics. 1995;29(2):311-322.

16. Stephens M, Smith NJ, Donnelly P. A new statistical method for haplotype reconstruction from population data. Am J Hum Genet. 2001;68(4):978-989.

17. Jhun E, He Y, Yao Y, Molokie RE, Wilkie DJ, Wang ZJ. Dopamine D3 receptor Ser9Gly and catechol-o-methyltransferase Val158Met polymorphisms and acute pain in sickle cell disease. Anesth Analg. 2014;119(5): 1201-1207.

18. Wang C, Cheng Y, Liu T, et al. A computational model for sex-specific genetic architecture of complex traits in humans: implications for mapping pain sensitivity. Mol Pain. 2008;4:13.

19. Bragdon EE, Light KC, Girdler SS, Maixner W. Blood pressure, gender, and parental hypertension are factors in baseline and poststress pain sensitivity in normotensive adults. Int J Behav Med. 1997;4(1):17-38.

20. Belfer I, Youngblood V, Darbari DS, et al. A GCH1 haplotype confers sex-specific susceptibility to pain crises and altered endothelial function in adults with sickle cell anemia. Am J Hematol. 2014;89(2):187-193.

21. Ittiwut $R$, Listman JB, Ittiwut $C$, et al. Association between polymorphisms in catechol-O-methyltransferase (COMT) and cocaine-induced paranoia in European-American and African-American populations. Am J Med Genet B Neuropsychiatr Genet. 2011;156B(6):651-660.

22. McLeod HL, Fang L, Luo X, Scott EP, Evans WE. Ethnic differences in erythrocyte catechol-O-methyltransferase activity in black and white Americans. J Pharmacol Exp Ther. 1994;270(1):26-29.

23. Bakshi N, Stinson J, Ross D, et al. Development, content validity and user review of a web-based multi-dimensional pain diary for adolescent and young adults with sickle cell disease. Clin J Pain. 2015;31(6):580-590.

24. A global reference for human genetic variation The 1000 Genomes Project Consortium. Nature; 526, 68-74 (01 October 2015). Available from: https://www.nature.com/articles/nature15393. Accessed January $17,2018$.
Journal of Pain Research

\section{Publish your work in this journal}

The Journal of Pain Research is an international, peer reviewed, open access, online journal that welcomes laboratory and clinical findings in the fields of pain research and the prevention and management of pain. Original research, reviews, symposium reports, hypothesis formation and commentaries are all considered for publication.

\section{Dovepress}

The manuscript management system is completely online and includes a very quick and fair peer-review system, which is all easy to use. Visit http://www.dovepress.com/testimonials.php to read real quotes from published authors. 